\title{
Two simple and reliable metrics of molecular planarity: Molecular planarity parameter (MPP) and span of deviation from plane (SDP)
}

\author{
Tian $\mathrm{Lu}$
}

Beijing Kein Research Center for Natural Sciences, Beijing 100022, P. R. China (http://www.keinsci.com)

E-mail: sobereva@sina.com

\section{Author information}

Tian Lu ORCiD: 0000-0002-1822-1229 E-mail: sobereva@sina.com

\begin{abstract}
Planarity is a very important structural character of molecules, which is closely related to many molecular properties. However, there is currently no simple, universal, and robust way to measure molecular planarity. In order to fill this evident gap, we propose two metrics of molecular planarity, namely molecular planarity parameter (MPP) and span of deviation from plane (SDP), to quantitatively characterize planarity of molecules. MPP reflects the overall degree of deviation of the structure from a plane, while SDP represents the span of the structural deviation relative to the fitting plane, respectively, they are complementary to each other. The examples in this article demonstrate that these metrics have strong rationality and practicality. They can not only be used to investigate the planarity of the entire molecule, but can also measure the planarity of local structures, and they can even be employed to study variation of molecular planarity during a dynamic process. In addition, we also proposes a new representation, namely coloring atoms according to their signed deviation distance to the fitting plane. This kind of map allows researchers to intuitively and quickly recognize position of the atoms in the system relative to the fitting plane. It can be seen
\end{abstract}


from the examples that this representation is very useful in graphically exhibiting molecular planarity. The methods proposed in this work have been implemented in our open-source analysis code Multiwfn, which can be freely obtained via http://sobereva.com/multiwfn. The use is very simple and rich file formats are supported as input file.

Keywords: Planarity; Multiwfn; Molecular structure; Annulene; Cyclo[18]carbon; Buckyball catcher 


\section{Introduction}

Planarity of a molecule is closely related to many molecular properties and thus is a key concept. For example, structural planarity influences mobility and ambipolarity of organic field-effect transistors (OFETs), ${ }^{[1]}$ degree of planarity of a molecule has correlation with its second harmonic generation efficiency, ${ }^{[2]}$ molecular aromaticity and $\pi$ conjugation are highly dependent of molecular planarity, ${ }^{[3]}$ a strong correlation has been reported between excitation energies and planarity of conjugated oligothiophenes. $^{[4]}$

Despite the importance of molecular planarity, there is still a lack of a simple and universal metric of molecular planarity. We noted that in literatures, the planarity is usually measured in terms of dihedrals. ${ }^{[1-4]}$ However, for a general system, how to choose appropriate dihedral(s) to unambiguously measure planarity is a problem. In this article, we will propose two simple, reliable, and universal metrics to characterize molecular planarity, namely molecular planarity parameter (MPP) and span of deviation from plane (SDP). In addition, we will show that deviation of atom positions over or below the fitting plane can be exhibited by properly coloring the atoms, this new representation makes graphical analysis of planarity very intuitive and convenient. The methods proposed in this work have been implemented in our open-source code Multiwfn, ${ }^{[5]}$ which can be freely obtained via http://sobereva.com/multiwfn.

In the rest of this article, we will describe the definition of MPP and SDP in Section 2, then the implementation of MPP and SDP in Multiwfn will be mentioned in Section 3. Four illustrative applications of MPP and SDP will be given in Section 4 to demonstrate their values. Finally, in section 5 we summarize this article.

\section{Definition of MPP and SDP}

Before calculating the MPP or SDP of a molecule, the plane fitted by the atoms should be obtained via the least square method, technically this is easy to realize. ${ }^{[6]}$ First, the coordinates of all atoms are organized as a $3 \times N_{\text {atom }}$ matrix and subtract out the 
geometry center from it, then performing singular value decomposition (SVD) ${ }^{[7]}$ for this matrix. The subroutine for SVD has been supported by wide variety of popular mathematical libraries such as LAPACK. ${ }^{[8]}$ Assume $\mathbf{u}$ to be the left singular vector (a column vector with dimension of 3 ) corresponding to the least singular value, then the parameters of the fitting plane $A x+B y+C z+D=0$ will be $A=u_{1}, B=u_{2}, C=u_{3}$, and in order to make the plane pass through geometry center $\left(x_{\mathrm{c}}, y_{\mathrm{c}}, z_{\mathrm{c}}\right)$ of the atoms, the $D$ is solved to be $-\left(u_{1} x_{\mathrm{c}}+u_{2} y_{\mathrm{c}}+u_{3} z_{\mathrm{c}}\right)$.

Once the fitting plane is obtained, then the molecular planarity parameter (MPP) can be calculated as root-mean-square of deviation of the atoms from the fitting plane, namely

$$
M P P=\sqrt{\frac{1}{N_{\text {atom }}} \sum_{i} d_{i}^{2}}
$$

where $d_{i}$ is the distance between atom $i$ and the fitting plane, and it can be easily evaluated as ${ }^{[9]}$

$$
d_{i}=\frac{\left|A x_{i}+B y_{i}+C z_{i}+D\right|}{\sqrt{A^{2}+B^{2}+C^{2}}}
$$

It is useful to distinguish whether an atom is above or below the fitting plane. We define signed distance of atom $i$ to the fitting plane as

$$
d_{i}^{\mathrm{s}}=\frac{A x_{i}+B y_{i}+C z_{i}+D}{\sqrt{A^{2}+B^{2}+C^{2}}}
$$

then the span of deviation from plane (SDP) can be calculated as follows

$$
S D P=d_{\max }^{\mathrm{s}}-d_{\min }^{\mathrm{s}}
$$

where $d_{\max }^{\mathrm{s}}$ and $d_{\min }^{\mathrm{s}}$ denote the most positive and most negative values of $d^{\mathrm{s}}$ among all considered atoms, respectively.

Obviously, MPP is a straightforward measure of the overall degree of deviation of the atoms from the fitting plane, while SDP aims at exhibiting deviation span of the system with respect to the fitting plane. Obviously, the smaller the two values, the more planar the system. However, the two metrics of planarity are complementary to each other, positive correlation between them is not always expected. 
We noticed that $d^{\mathrm{s}}$ has another use. If atoms in a molecular structure map are colored according to the value of $d^{\mathrm{s}}$, then the relative position of the atoms with respect to the fitting plane can be displayed very clearly. As will be seen from the examples later, this new representation is quite helpful in graphically exhibiting molecular planarity in practical research.

\section{Implementation of MPP and SDP in Multiwfn}

Evaluation of MPP and SDP has been supported by our freely available code Multiwfn $^{[5]}$ since version 3.8. Multiwfn accepts almost all known structure files of chemical systems for this kind of calculation, for example, xyz, pdb, pqr, mol, mol2, cif, gro, and so on. Executable files of Multiwfn for all platforms are directly available from its website, including Windows, Linux and MacOS.

The process of using Multiwfn to calculate MPP and SDP is extremely simple. After booting up Multiwfn and load input file, user just needs to input "MPP" in the main menu, and input the indices of the atoms for which the MPP and SDP will be calculated, then intermediate information yielded during evaluation of MPP and SDP as well as the final result will be immediately printed, Fig. 1 is an example of the output. It is worth to emphasize that MPP and SDP are not necessarily to be calculated for the whole system, one can evaluate them for any part of the system to study planarity of a local region by inputting corresponding atom indices in Multiwfn.

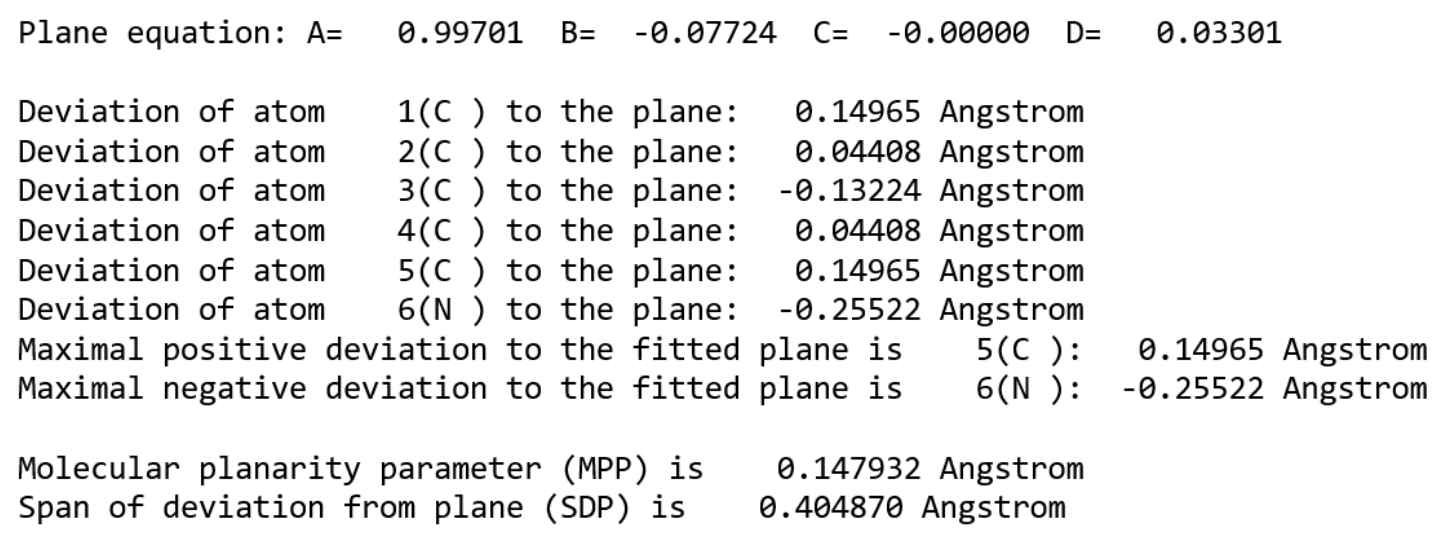

Fig. 1 Example of calculation output of MPP and SDP in Multiwfn. 
After finishing the calculation of MPP and SDP, Multiwfn will ask user if outputting a pqr file, ${ }^{[10]}$ in which the "charge" atomic property corresponds to the $d^{\text {s }}$ value. By loading this file into VMD visualization software, ${ }^{[1]}$ one can color atoms according to $d^{\text {s }}$ by setting "Coloring Method" as "Charge" in "Graphics Representation" panel, then fluctuation of atoms around the fitting plane can be vividly visualized.

Multiwfn is not only able to calculate MPP and SDP for a single geometry, but can also calculate them for specific frames of a trajectory recorded in xyz format. After entering the function for calculating MPP and SDP, if the input file is detected to be a multiple frame xyz file, then user will be asked to input the indices of the frames to be calculated. After that, frame index, MPP and SDP will be exported as the three columns of a plain text file. This feature is very useful to study evolution of molecular planarity over molecular dynamics simulation process or over intrinsic reaction path (IRC) of a chemical process.

\section{Illustrative application of MPP and SDP}

In this section, a few examples will be given to illustrate reliability and practical value of MPP and SDP, as well as the usefulness of coloring atoms according to $d^{\mathrm{s}}$. Unless otherwise specified, the geometries were obtained by geometry optimization using Gaussian 16 A.03 program, ${ }^{[12]} \omega \mathrm{B} 97 \mathrm{XD}$ exchange-correlation functional ${ }^{[13]}$ in combination with def2-TZVP basis set $^{[14]}$ was employed in the calculation. No imaginary frequency was found for the structures. All data were calculated by Multiwfn $3.8(\mathrm{dev}),{ }^{[5]}$ all molecular structure maps were plotted by VMD 1.9.3. ${ }^{[11]}$

\section{1 [n]annulene of different sizes}

$[n]$ annulene is a kind of unsubstituted monocyclic hydrocarbons and has received wide investigations. ${ }^{[15,16]}$ When $n$ is even, the general formula is $\mathrm{C}_{n} \mathrm{H}_{n}$. Some $[n]$ annulenes are purely planar while some are not. In order to characterize planarity of 
the $[n]$ annulene series, we calculated MPP and SDP for carbon atoms of $[n]$ annulene ranging from $n=4$ to 22, only the case of even $n$ was taken into account. In addition, the atoms are colored according to $d^{\mathrm{s}}$ to clearly exhibit their relative location with respect to the plane fitted for the ring. The data and maps are collectively shown in Fig.

2.

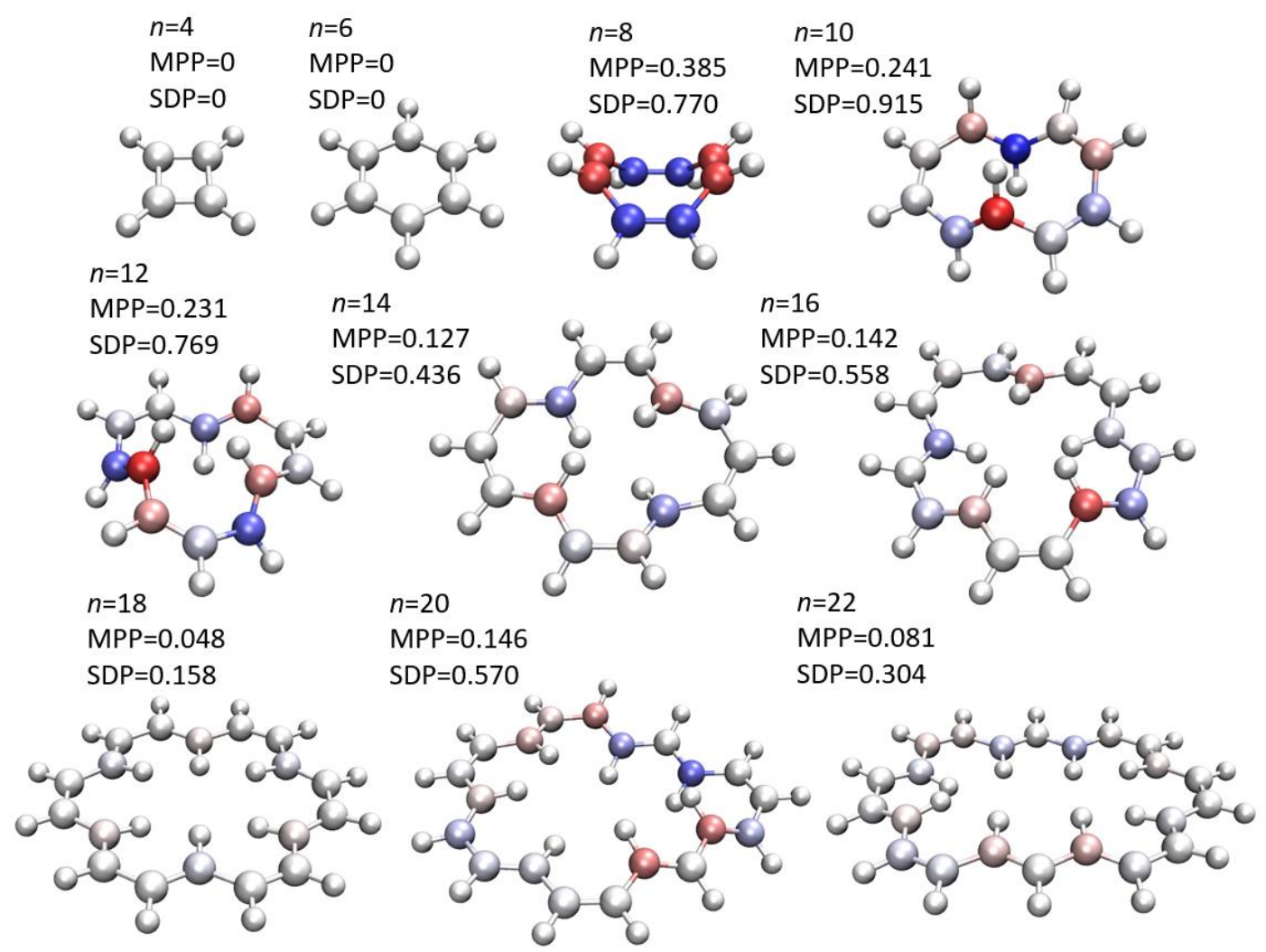

Fig. 2 MPP and SDP (in $\AA$ ) calculated for all carbons of $[n]$ annulene optimized at $\omega \mathrm{B} 97 \mathrm{XD} / \mathrm{def} 2-\mathrm{TZVP}$ level. Atoms are colored according to $d^{\mathrm{s}}$ values using color scale from $0.5 \AA$ (blue) to $+0.5 \AA$ (red). The bluer (redder) the color, the larger the distance of the atom below (above) the fitting plane.

From Fig. 2, one can find that MPP and SDP successfully quantified the planarity of the $[n]$ annulenes, their values are very consistent with the degree of deviation of the system from a plane that can be judged from the structure maps. Among all the considered molecules, [8] annulene has the largest MPP; indeed, from the structure map one can see that its overall shape is least like a plane. [10]annulene does not have largest MPP, but have highest SDP, indicating that there must be two or more atoms whose 
positions are extremely deviated from the plane fitted for the entire ring.

It is evident from Fig. 2 that coloring the atoms according to $d^{\text {s }}$ makes relative position of the atoms with respect to the fitting plane easily recognizable. The atoms that are approximately on the fitting plane show a white color, while the atoms obviously lying above and below the plane are displayed as blue and red, respectively. In the map of [10]annulene, the reddest and bluest atoms directly correspond to the two atoms actually used to calculate SDP, as they deviate from the fitting plane most respectively from two opposite directions.

\subsection{Dynamics of cyclo[18]carbon}

MDP and SDP can also be used to characterize variation of planarity during a dynamic process. As an example, in this section we will study MDP and SDP along the ab-initio molecular dynamics trajectory of cyclo[18]carbon at $298.15 \mathrm{~K}$. This trajectory was taken from our previous work and simulation details can be found in Ref. [17]. It is worth to note that the cyclo[18]carbon is an all-carboatomic ring experimentally observed recently, ${ }^{[18]}$ since then, it has aroused widespread interest among theoretical chemists, and we have recently conducted a lot of research on this system. ${ }^{[17,19-24]}$ The variation curves of MDP and SDP of the cyclo[18]carbon dynamics trajectory are plotted in Fig. 3. Although the trajectory file has as many as 2000 frames, since the code used to evaluate MDP and SDP in Multiwfn is efficient and the algorithm is simple, the calculation for all frames takes less than one second. 


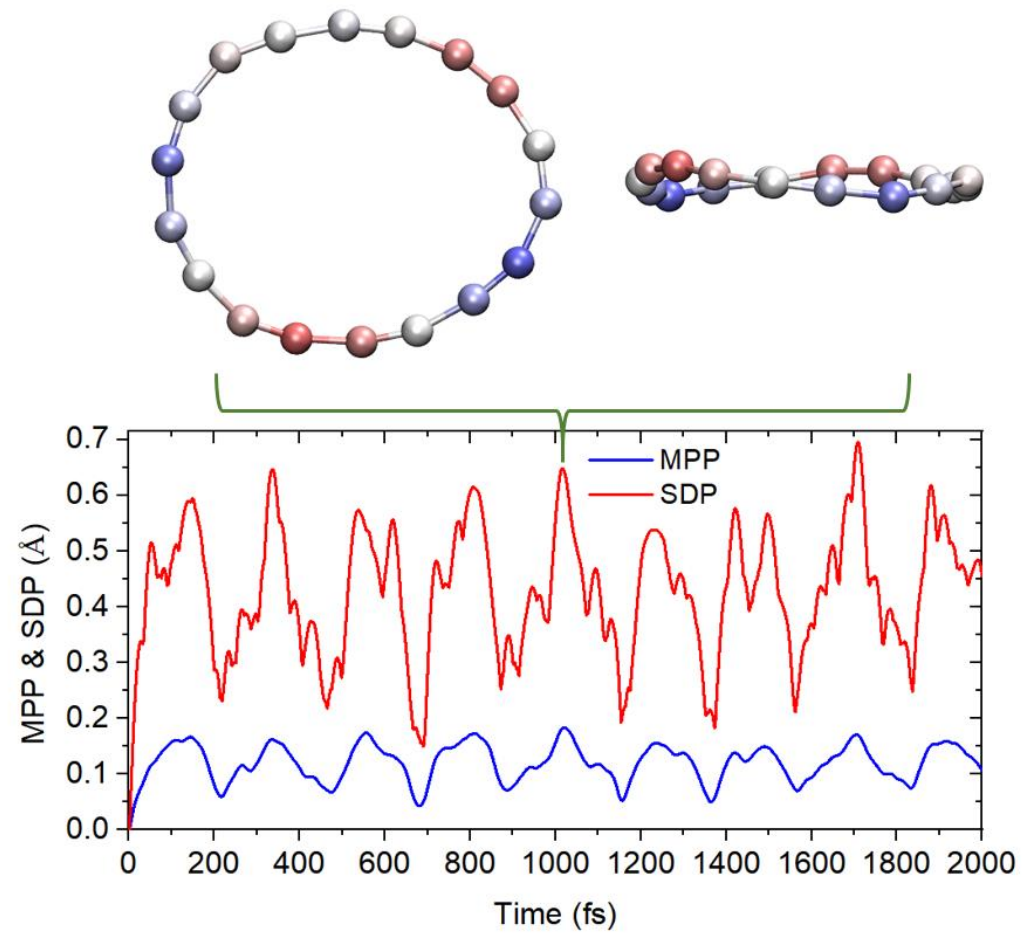

Fig. 3 Variation of MPP and SDP during molecular dynamics process of cyclo[18]carbon at $298.15 \mathrm{~K}$. The initial geometry corresponds to minimum on potential energy surface and is exactly planar. The snapshot of two different perspectives at $1015 \mathrm{fs}$ is shown at the top, atoms are colored according to $d^{\mathrm{s}}$ values using color scale from $-0.5 \AA$ (blue) to $+0.5 \AA$ (red).

We have shown that cyclo[18]carbon has a large flexibility. Although it is exactly planar at minimum of potential energy surface, ${ }^{[23]}$ the perfect planarity can be easily broken due to atomic thermal motion perpendicular to the ring. ${ }^{[17]}$ From the MPP and SDP shown in Fig. 3, this point is very clearly exhibited; it can be seen that in most of the time, the values of MPP and SDP are relatively large, reflecting that this molecule lacks of ideal planar character in the real environment. It can also be found that the two planarity metrics fluctuate regularly with period of approximately $220 \mathrm{fs}$, and their variations have an evident positive correlation. The molecular structure map corresponding to 1015 fs colored by the $d^{\text {s }}$ values in Fig. 3 is evidently helpful for intuitively understanding the planarity of the cyclo[18]carbon. As shown by the colors, the atoms below and above the ring plane alternately appear.

\subsection{Buckyball catcher host-guest complex}

Buckyball $\mathrm{C}_{60}$ can be stably caught by buckyball catcher via its two corannulene 
pincers. ${ }^{[25]}$ In order to illustrate the feasibility of using MPP and SDP to study planarity of substructures of an entire system, the buckyball catcher host-guest complex will be taken as example. Its theoretical geometry was taken from Ref. [26] and plotted as Fig. 4. We calculated MPP and SDP for the carbons in the two corannulene fragments separately. Because this system is fully symmetric, the values for the two fragments are exactly identical, namely MPP $=0.352 \AA$ and $\mathrm{SDP}=0.883 \AA$. Since these values deviate from zero markedly, though the corannulene fragment visually shows some degree of planarity, the planarity is relative limited. In Fig. 4 we colored carbon atoms in the corannulene pincers according to the $d^{\mathrm{s}}$ values respectively calculated for the two parts, according to the colors one can very intuitively distinguish the atoms in the concave area and those in the edge-up area.

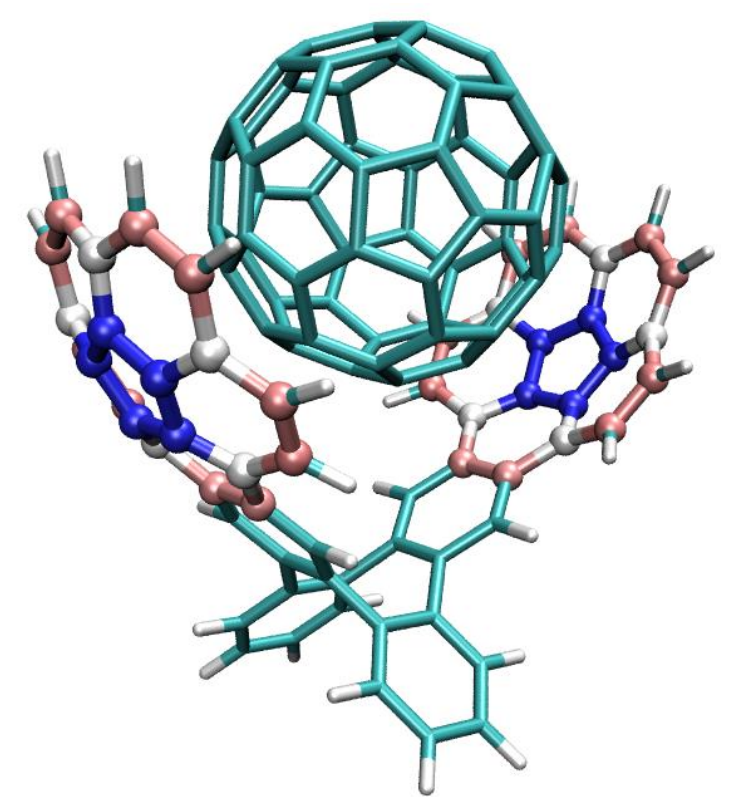

Fig. 4 Buckyball catcher host-guest complex. The carbon atoms in the two corannulene fragments are colored according to $d^{\mathrm{s}}$ values with color scale from - $0.6 \AA$ (blue) to $+0.6 \AA$ (red). Note that the $d^{\mathrm{s}}$ values are separately calculated for the two fragment.

\subsection{Molecular crystal of tetrathiafulvalene derivative}

MPP, SDP and the representation of coloring atoms by $d^{\mathrm{s}}$ can also be applied to crystal, an example is given in this section. Rovira et al. reported crystal of bis(4,5- 
dihydronaphto[1,2-d])tetrathiafulvalene (BDHN-TTF), ${ }^{[27]}$ it is found that the BDHNTTF is nonplanar. A supercell of BDHN-TTF was constructed by Multiwfn based on the cif file provided in the reference [27], see Fig. 5. In order to quantitatively characterize the planarity, we calculated MPP and SDP of all non-hydrogen atoms for the molecule at the center of the supercell, the values are 0.239 and $0.796 \AA$, respectively. Since the MPP is not particularly large, it shows that BDHN-TTF possess a moderate planarity. However, the large SDP implies that there are regions with significant distortion with respect to the plane fitted for the whole molecule. In Fig. 5, the non-hydrogen atoms of the central BDHN-TTF molecule are colored according to their $d^{\mathrm{s}}$ values, it can be quickly identified that the most severe distortion from the fitting plane comes from the $s p^{3}$ hybridized carbons, as their colors are either reddest or bluest.

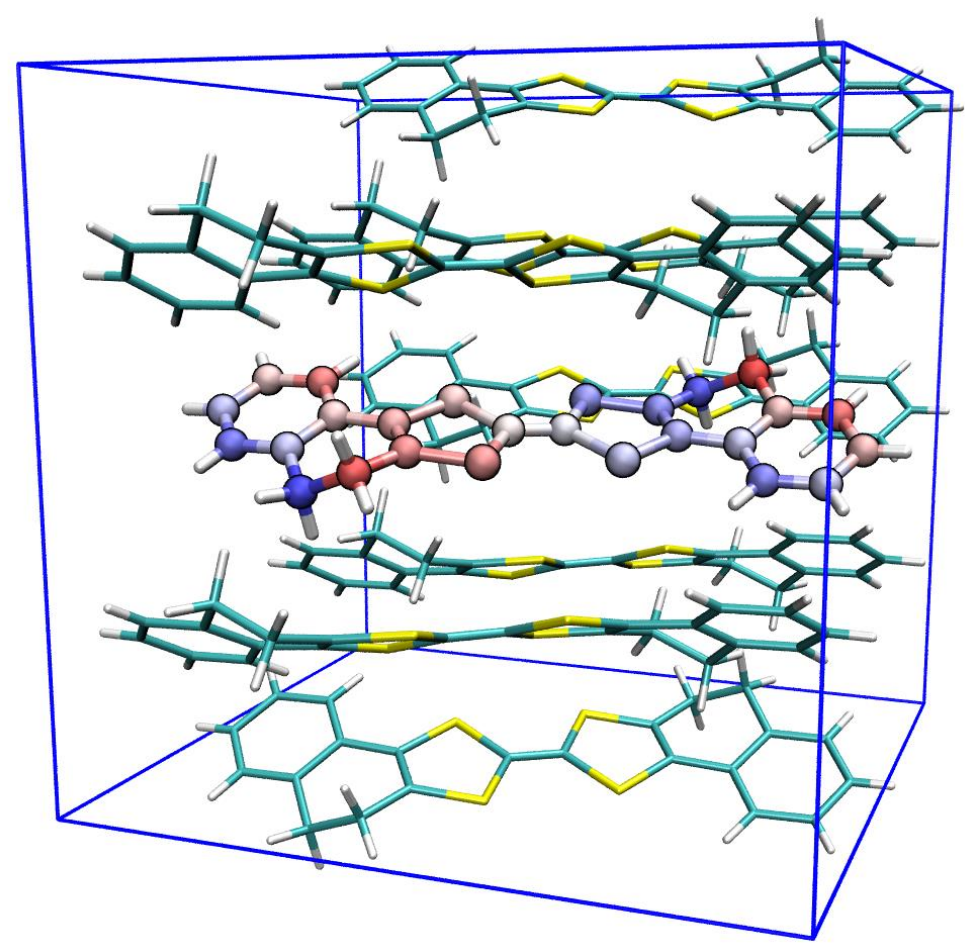

Fig. 5 BDHN-TTF molecular crystal. The non-hydrogen atoms in the central molecule are colored according to $d^{\mathrm{s}}$ values using color scale from $-0.5 \AA$ (blue) to $+0.5 \AA$ (red).

\section{Summary}

Planarity is an important structural property especially for $\pi$-conjugated molecules, 
which usually show planar or quasi-planar configuration. In this work, we proposed molecular planarity parameter (MPP) and span of deviation from plane (SDP) for quantitatively characterize planarity of a whole molecule or its substructures. The former reflects the overall degree of deviation of the structure from a plane, while the latter measures the span of the structure deviates to the fitting plane. Examples in this article illustrated that these two metrics of planarity are useful, reliable, and universal, and they are complementary to each other in characterizing planarity. In addition, coloring atoms according to their signed deviations to fitting plane is suggested, this new representation makes structural character about planarity quite intuitive, one can conveniently identify relative positions of atoms with respect to the fitting plane. Since the method proposed in this work makes quantitative and visual analysis of molecular planarity quite easy, and they have been implemented into the popular and freely available code Multiwfn, we expect that the analysis method in this article will be widely employed when researchers investigate molecular planarity.

\section{Acknowledgments}

This research was not funded.

\section{Conflicts of interest}

The authors declare no conflict of interest.

\section{References}

1. D.-H. Lim; S.-Y. Jang; M. Kang; S. Lee; Y.-A. Kim; Y.-J. Heo; M.-H. Lee; D.-Y. Kim. A systematic study on molecular planarity and D-A conformation in thiazolothiazole- and thienylenevinylene-based copolymers for organic field-effect transistors. J. Mater. Chem. C, 5, 10126-10132 (2017). DOI: 10.1039/C7TC02273E.

2. A. P. Menezes; A. Jayarama; S. W. Ng. Crucial role of molecular planarity on the second order nonlinear optical property of pyridine based chalcone single crystals. J. Mol. Struct., 1088, 85-94 (2015). DOI: 10.1016/j.molstruc.2015.02.011.

3. B. M. Wood; A. C. Forse; K. A. Persson. Aromaticity as a Guide to Planarity in Conjugated Molecules and Polymers. J. Phys. Chem. C, 124, 5608-5612 (2020). DOI: 10.1021/acs.jpcc.0c01064. 
4. J. Sjöqvist; J. Maria; R. A. Simon; M. Linares; P. Norman; K. P. R. Nilsson; M. Lindgren. Toward a Molecular Understanding of the Detection of Amyloid Proteins with Flexible Conjugated Oligothiophenes. J. Phys. Chem. A, 118, 9820-9827 (2014). DOI: 10.1021/jp506797j.

5. T. Lu; F. Chen. Multiwfn: A Multifunctional Wavefunction Analyzer. J. Comput. Chem., 33, 580592 (2012). DOI: 10.1002/jcc.22885.

6. joriki. Best Fitting Plane given a Set of Points, https://math.stackexchange.com/q/99317 (accessed on Jun 4, 2021)

7. Wikipedia. Singular value decomposition, https://en.wikipedia.org/wiki/Singular_value decomposition (accessed on Jun 4, 2021)

8. LAPACK - Linear Algebra PACKage, http://www.netlib.org/lapack/index.html (accessed on Jun 4, 2021)

9. Wikipedia. Plane (geometry), https://en.wikipedia.org/wiki/Plane (geometry) (accessed on Jun 4, 2021)

10. PQR biomolecular structure format, https://www.ics.uci.edu/ dock/manuals/apbs/html/userguide/a2566.html (Accessed on Jun 5, 2021).

11. W. Humphrey; A. Dalke; K. Schulten. VMD: Visual molecular dynamics. J. Mol. Graph., 14, 3338 (1996). DOI: 10.1016/0263-7855(96)00018-5.

12. M. J. Frisch; G. W. Trucks; H. B. Schlegel; G. E. Scuseria; M. A. Robb; J. R. Cheeseman; G. Scalmani; V. Barone; G. A. Petersson; H. Nakatsuji; X. Li; M. Caricato; A. V. Marenich; J. Bloino; B. G. Janesko; R. Gomperts; B. Mennucci; H. P. Hratchian; J. V. Ortiz; A. F. Izmaylov; J. L. Sonnenberg; Williams; F. Ding; F. Lipparini; F. Egidi; J. Goings; B. Peng; A. Petrone; T. Henderson; D. Ranasinghe; V. G. Zakrzewski; J. Gao; N. Rega; G. Zheng; W. Liang; M. Hada; M. Ehara; K. Toyota; R. Fukuda; J. Hasegawa; M. Ishida; T. Nakajima; Y. Honda; O. Kitao; H. Nakai; T. Vreven; K. Throssell; J. A. Montgomery Jr.; J. E. Peralta; F. Ogliaro; M. J. Bearpark; J. J. Heyd; E. N. Brothers; K. N. Kudin; V. N. Staroverov; T. A. Keith; R. Kobayashi; J. Normand; K. Raghavachari; A. P. Rendell; J. C. Burant; S. S. Iyengar; J. Tomasi; M. Cossi; J. M. Millam; M. Klene; C. Adamo; R. Cammi; J. W. Ochterski; R. L. Martin; K. Morokuma; O. Farkas; J. B. Foresman; D. J. Fox. Gaussian 16 A.03, Wallingford, CT, 2016. 13. J.-D. Chai; M. Head-Gordon. Long-range corrected hybrid density functionals with damped atomatom dispersion corrections. Phys. Chem. Chem. Phys., 10, 6615-6620 (2008). DOI: 10.1039/b810189b. 14. F. Weigend; R. Ahlrichs. Balanced basis sets of split valence, triple zeta valence and quadruple zeta valence quality for $\mathrm{H}$ to Rn: Design and assessment of accuracy. Phys. Chem. Chem. Phys., 7, 3297-3305 (2005). DOI: 10.1039/b508541a.

15. C. S. Wannere; K. W. Sattelmeyer; H. F. Schaefer III; P. v. R. Schleyer. Aromaticity: The Alternating C-C Bond Length Structures of [14]-, [18]-, and [22]Annulene. Angew. Chem. Int. Ed., 43, 4200-4206 (2004). DOI: https://doi.org/10.1002/anie.200454188.

16. E.-K. Mucke; B. Schönborn; F. Köhler; R. Herges. Stability and Aromaticity of Charged Möbius[4n]Annulenes. J. Org. Chem., 76, 35-41 (2011). DOI: 10.1021/jo100798e.

17. Z. Liu; T. Lu; Q. Chen. Vibrational spectra and molecular vibrational behaviors of all-carboatomic rings, cyclo[18]carbon and its analogues. Chem. - Asian J., 16, 56 (2021). DOI: 10.1002/asia.202001228. 18. K. Kaiser; L. M. Scriven; F. Schulz; P. Gawel; L. Gross; H. L. Anderson. An sp-hybridized molecular carbon allotrope, cyclo[18]carbon. Science, 365, 1299-1301 (2019).

19. T. Lu; Q. Chen. Ultrastrong Regulation Effect of the Electric Field on the All-Carboatomic Ring Cyclo[18]Carbon. ChemPhysChem, 22, 386-395 (2021). DOI: 10.1002/cphc.202000903.

20. Z. Liu; T. Lu; Q. Chen. Comment on "Theoretical investigation on bond and spectrum of 
cyclo[18]carbon (C18) with sp-hybridized". J. Mol. Model., 27, 42 (2021). DOI: 10.1007/s00894-02104665-9.

21. Z. Liu; T. Lu; Q. Chen. Intermolecular interaction characteristics of the all-carboatomic ring, cyclo[18]carbon: Focusing on molecular adsorption and stacking. Carbon, 171, 514-523 (2021). DOI: 10.1016/j.carbon.2020.09.048.

22. Z. Liu; T. Lu; Q. Chen. An sp-hybridized all-carboatomic ring, cyclo[18]carbon: Electronic structure, electronic spectrum, and optical nonlinearity. Carbon, 165, 461-467 (2020). DOI: 10.1016/j.carbon.2020.05.023.

23. Z. Liu; T. Lu; Q. Chen. An sp-hybridized all-carboatomic ring, cyclo[18]carbon: Bonding character, electron delocalization, and aromaticity. Carbon, 165, 468-475 (2020). DOI: 10.1016/j.carbon.2020.04.099.

24. T. Lu; Q. Chen; Z. Liu. A thorough theoretical exploration of intriguing characteristics of cyclo[18]carbon: Geometry, bonding nature, aromaticity, weak interaction, reactivity, excited states, vibrations, molecular dynamics and various molecular properties. ChemRxiv (2019). DOI: 10.26434/chemrxiv.11320130.

25. M. A. Blood-Forsythe; T. Markovich; R. A. DiStasio; R. Car; A. Aspuru-Guzik. Analytical nuclear gradients for the range-separated many-body dispersion model of noncovalent interactions. Chem. Sci., 7, 1712-1728 (2016). DOI: 10.1039/C5SC03234B.

26. R. Sure; S. Grimme. Comprehensive Benchmark of Association (Free) Energies of Realistic HostGuest Complexes. J. Chem. Theory Comput., 11, 3785-3801 (2015). DOI: 10.1021/acs.jctc.5b00296.

27. A. Campos; N. Oxtoby; S. Galindo; R. Pfattner; J. Veciana; S. T. Bromley; C. Rovira; M. MasTorrent. Structural and electronic characterisation of $\pi$-extended tetrathiafulvalene derivatives as active components in field-effect transistors. CrystEngComm, 18, 6149-6152 (2016). DOI: 10.1039/C6CE01200K. 